\title{
Structure Model for the Cooperative Domain in Ferroelectric Transition of Poly(vinylidene fluoride) Form I
}

\author{
Yasuhiro TAKAHASHI \\ Department of Macromolecular Science, \\ Graduate School of Science, Osaka University, \\ Toyonaka, Osaka 560-0043, Japan
}

(Received October 19, 2000; Accepted December 12, 2000)

KEY WORDS Poly(vinylidene fluoride) / Form I / Domain Structure / Ferroelectric Transition /

The previous papers showed that in crystal phase transitions of crystalline polymers ${ }^{1-3}$ including ferroelectric transitions, domain structures play important roles. The antiphase domain structure was found in form II of poly(vinylidene fluoride $)^{4}$ and satisfactorily explains phase transitions from form II to form III $^{1}$ via streak II and from form II to polar $\mathrm{II}^{2}$ in poly(vinylidene fluoride). The antiphase domain structure in form II is formed by two domains of antipolar structures that shift by $1 / 2 \mathrm{a}$ and $1 / 2 \mathrm{~b}$ bounded by structure parallel to the acplane. For phase transitions from form II to form III and from form II to polar II, the polar structure existing at the domain boundary grows in the direction of the $b$-axis and forms the polar structures of form III and polar II. In form II, the polar structure exists as a domain boundary, i.e., in disorder. On the other hand, glide type disorders were found in form I of poly(vinylidene fluoride) ${ }^{5}$ and were parallel to (010) and (110) planes (Figures 1 and 2).

Poly(vinylidene fluoride) form I is a ferroelectric polymer, in the crystalline region of which molecular orientation changes by $180^{\circ}$ reversal under an electricfield..$^{3,6}$ During transition, there exists region in which a group

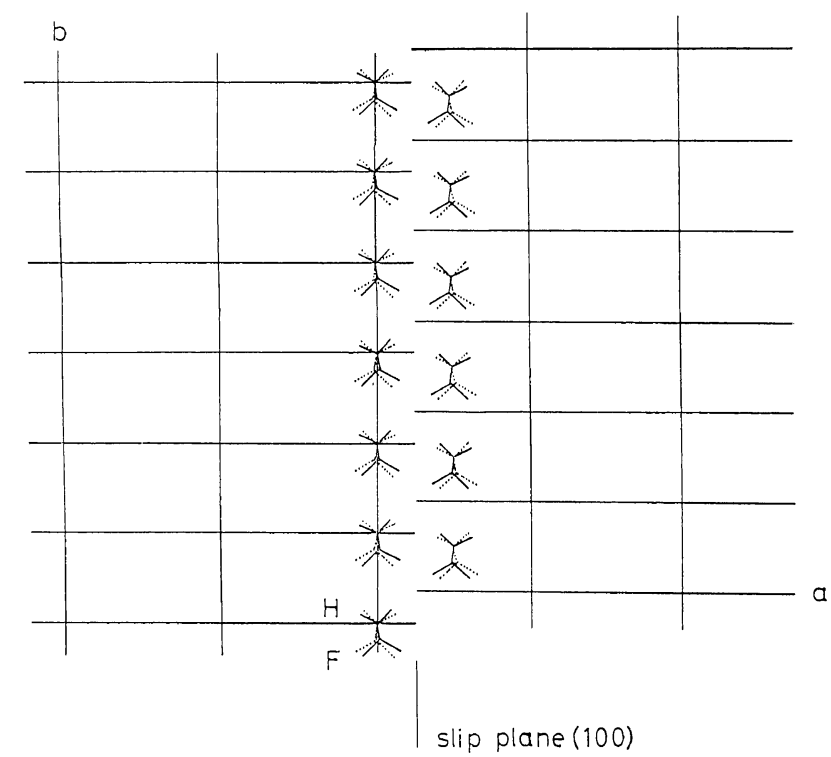

Figure 1. Schematic representation of glide type disorders parallel to (010) planes in poly(vinylidene fluoride) form I. of molecules moves cooperatively. This is the domain structure. The domain structure should posses an energetically stable surface. Form II domains in the antiphase domain structure are surrounded by the domain boundary parallel to (010) planes in disorder. Accordingly, in form I, the domain structure is considered surrounded by glide type disorders. The cooperative domain structure in form $I$ is surrounded by glide type disorders parallel to (110) and (010) planes. The domain structure is considered to grow by the cooperative motion of the molecules situated parallel to (110) and (010) planes. The shape of the domain structure in form $I$ is thus that of a pseudo-hexagon as shown in Figure 3.

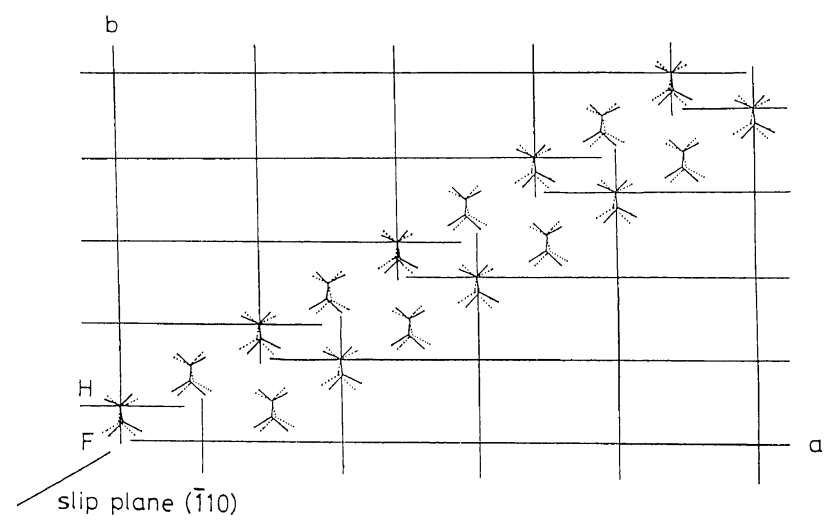

Figure 2. Schematic representation of glide type disorders parallel to (110) planes in poly(vinylidene fluoride) form I.

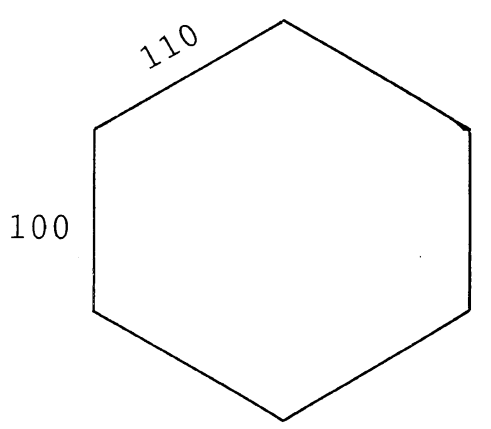

Figure 3. Structure model for the domain in which $180^{\circ}$ reversal motion cooperatively gives rise to molecules. 


\section{REFERENCES}

1. Y. Takahashi, Polymer J., 15, 733 (1983).

2. Y. Takahashi, T. Ishida, and M. Furusaka, J. Polym. Sci. Polym. Phys. Ed., 26, 2267 (1988).
3. Y. Takahashi, J. Macromol. Sci. Phys., B37, 421 (1998).

4. Y. Takahashi and H. Tadokoro, Macromolecules, 16, 1880 (1983).

5. Y. Takahashi, Macromolecules, 26, 1471 (1993).

6. Y. Takahashi, Y. Nakagawa, H. Miyaji, and K. Asai, J. Polym. Sci. Polym. Lett. Ed., 25, 153 (1987). 